\title{
Una opción para el mejoramiento de las operaciones aéreas
}

\section{TO Rafael Pérez Uribe, docente IMA}

\author{
MY. Marcos Manuel Guzmán Alfaro, (Compiladores)*
}

En alguna oportunidad el comandante de una base de combate, en su angustia por administrar mejor los recursos a su cargo, se preguntó: ¿Cuáles son las herramientas gerenciales necesarias para administrar efectivamente el personal de pilotos, de tal manera que se asegure el éxito en la planeación, dirección y ejecución de las operaciones aéreas y se garantice su desarrollo dentro de parámetros de calidad, de manera permanente?

Una pregunta con respuesta dificil, que seguramente se viene tratando de responder desde hace mucho tiempo en el ámbito de la macro y microeconomía desde famosos economistas como Adam Smith y David Ricardo; sociólogos como Max Weber y Mary Parker Follet; eminentes ingenieros como Frederick Taylor y sus seguidores; empresarios gerentes como Henry Fayol y sus seguidores desde el siglo pasado, hasta los Gurus gerenciales actuales como Peter Drucker, Kenichi Ohmae y Michael Porter, para mencionar solo algunos de ellos.

El siguiente escrito es un intento para expresar una de las posibles respuestas a esa complicada pregunta a la luz del aporte de dos oficiales de la Fuerza Aérea Colombiana, que presentan un sistema para administrar las operaciones aéreas, basado en el crecimiento humano del personal involucrado en términos de calidad, dirigido al mejoramiento continuo de los procesos críticos que intervienen en estas actividades. La implantación de un sistema de gerencia de calidad fruto del trabajo de esta investigación es fórmula válida que permite alcanzar condiciones de conformidad con la Norma ISO 9001:2000 y al tiempo establecer el ambiente organizacional adecuado para la gestión humana (especialmente de pilotos) en la dirección, planeación y ejecución de operaciones aéreas. Esta propuesta es sencilla de comprender y aplicar, pero requiere del compromiso de la alta dirección de cada Unidad de optimizar el talento humano, técnico y material a su alcance para mejorar la efectividad en los resultados sin mayores gastos o cambios, aparte de aquellos que se requieran en la actitud de comandantes y pilotos para facilitar la implantación de herramientas gerenciales modernas y novedosas. Con esta idea en mente, la propuesta de un sistema de calidad está enfocada a la realidad de los pilotos de UH-60 de un Comando Aéreo de Combate que ha desempeñado un papel preponderante en el desarrollo de las operaciones dentro del conflicto interno del pais con su participación directa en misiones de aplicación y multiplicación de la fuerza en condiciones diurnas y nocturnas.

En el diario operar, se ha podido observar que a pesar de la amplia experiencia operativa que respalda el éxito en la ejecución de las misiones, han surgido situaciones que afectan la calidad del servicio prestado, clasificadas en cinco grandes grupos para facilitar su investigación y análisis: las condiciones generales del servicio de

* Compilado del trabajo de grado presentado por los Mayores Javier Iván Delgado Garzón y Juan Carlos Puentes Mora. Propuesta de Implantación de un Sistema de Calidad para Maximizar el Desempeño del Personal de Pilotos de UH-60 de un Comando Aéreo de Combate de la Fuerza Aérea Colombiana. Fuerza Aérea Colombiana. Instituto Militar Aeronáutico. Bogotá, D.C. 2003. 


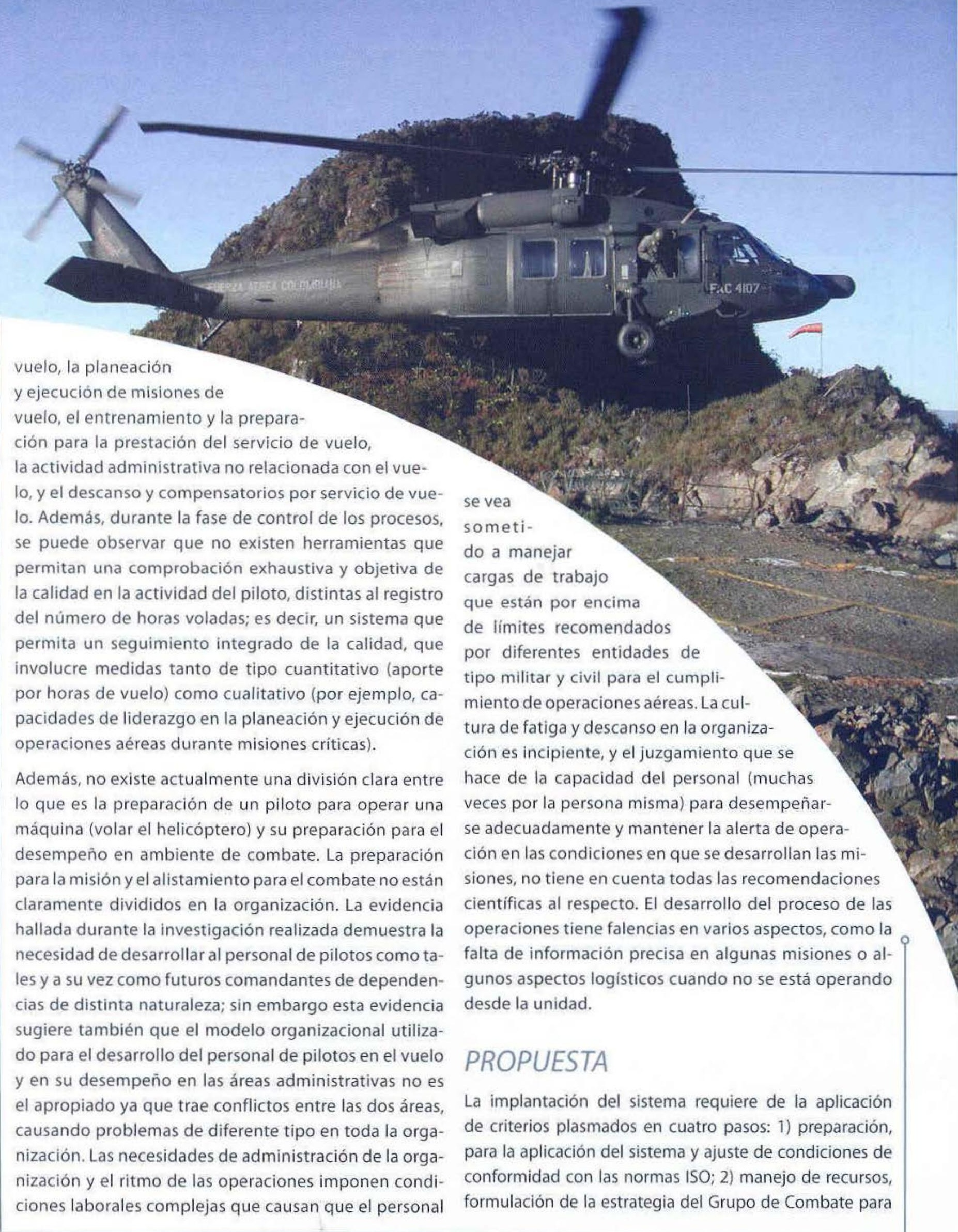


aprovechar de la mejor forma el talento y potencial de pilotos; 3) realización o cumplimiento de la misión como tal (operaciones aéreas) y 4) mejoramiento continuo, utilizando estrategias de gestión humana para lograr el objetivo principal del trabajo junto con aplicación de criterios ISO para establecer condiciones de conformidad de certificación en la ejecución de operaciones, de ser necesario.

\section{1) PREPARACIÓN PARA EL DESARROLLO DEL SISTE-}

MA. Hay tres pasos en esta preparación. El primero lo constituye el establecimiento de los principios, el concepto y el enfoque que se le dará a todo el esfuerzo que está a punto de acometer la organización en la búsqueda de la implantación del sistema de calidad. El segundo es cumplir con un mínimo de requisitos generales y de documentación. La mayoría está constituido por documentos ya existentes, de los que se debe asegurar su aplicabilidad y actualización, y la aplicación de algunas herramientas y estrategias que hacen parte de los aspectos a definir antes de adelantar la implantación del sistema de calidad. De entre una extensa gama, algunas de las herramientas propuestas son gráficas de Pareto para detectar errores, problemas o defectos; flujogramas y diagramas de causa y efecto para visualizar los eventos de los procesos; implementación de un sistema de sugerencias y de reconocimientos. El tercer paso se refiere a las condiciones de implantación del sistema que parten del Comandante de la Unidad y posteriormente de todos los demás niveles del Comando de Combate, con lo cual se asegura el éxito del sistema, formulando la política de calidad y comprometiendo a los demás niveles de la organización.

2) MANEJO DE RECURSOS. Se creó un modelo de gestión para el personal de pilotos, a partir de los hallazgos de esta investigación y los diferentes criterios de gestión humana que se coloca a disposición de los niveles de decisión como parte de la propuesta de implantación del sistema de calidad. Es importante adelantar las actividades necesarias para subsanar las falencias encontradas a lo largo de la investigación (ver Cuadro 1).
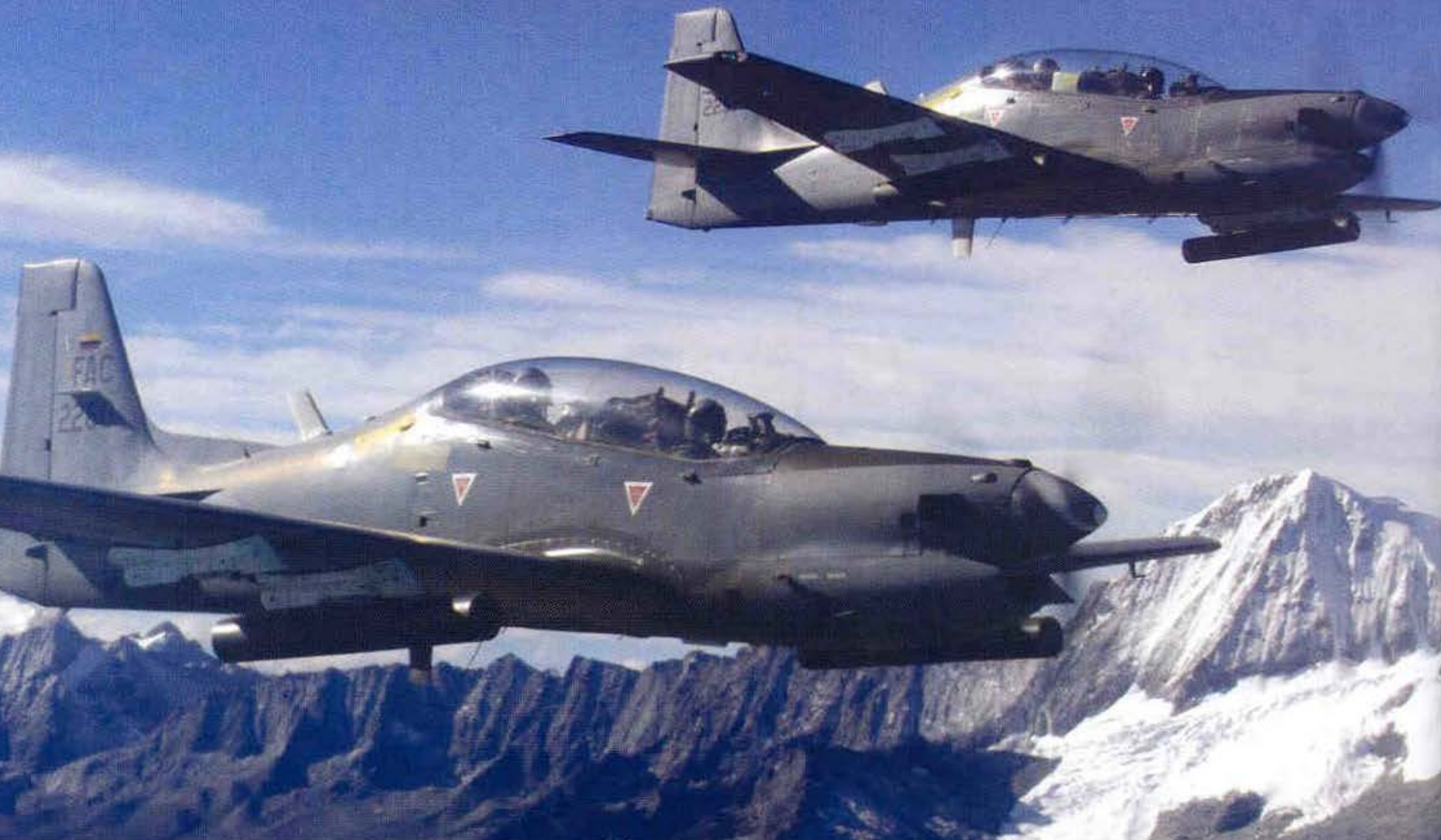
PROCESO DEINDUCCIÓN

Los pilotos recién asignados no son sometidos a procesos de inducción como es recomendado por expertos.
Abarcar parte del programa de entrenamiento continuado antes de someter a los nuevos pilotos al entorno de misión.

\section{ENTRENAMIENTO PARA LA MISIÓN}

No hay diferencia clara entre los niveles de Incorporar un nivel o reglamentar lo respectivo para entrenamiento inicial y de misión.
El entrenamiento de control anual nollena expectativas de entrenamiento de misión. incluir entrenamiento de misión.

Dejar el control anual como está, ya que su propósito es diferente e implementar una fase de misión o avanzada estandarizada.

Existen tres unidades de la FAC operando UH-60 y no existe un programa formal que entrene por igual a los pilotos.
Incorporar programas de entrenamiento que abarquen a todos los escuadrones que operan el equipo.

\section{DESARROLLO DE PERSONAL (PROYECCIONN)}

La programación de las misiones de vuelo dependen en gran parte de factores ajenos al Comando de Combate.

Es dificil determinar y distribuir la cantidad de horas voladas por cada piloto.

Los mínimos establecidos de horas de vuelo por mes calendario no satisfacen las necesidades de progresión en la carrera.
Establecer una metodología que asegure que los pilotos vuelen un determinado número de horas como parte de la proyección.

Establecer mínimos y estándares que mantengan el entrenamiento del personal para la misión y su progresión en la carrera como piloto.

\section{RECONOCIMIENTO DEL PERSONAL.}

Existe la idea entre algunos pilotos de que su trabajo no es reconocido justamente.
Elevar estas críticas y proponer soluciones a la alta gerencia. Revisar y proponer criterios de selección para otorgamiento de estimulos.

\section{RETRIBUCION ECONÓMICA (VIÁTICOS)}

Este tipo de compensación se ha constituido en aliciente para el personal, perolos reglamentos impiden su cobro en ciertas condiciones.
Sugerir un cambio en las reglamentaciones respectivas. Proponer que la prima de vuelo no esté atada al cumplimiento de un mínimo de horas de vuelo mensual sino a una certificación de idoneidad.

\section{AMBIENTE DE TRABAJO}

La gran mayoría del personal opina que el proceso de planeación para la misión tiene fallas en cuanto a la falta de información.

Según el personal de pilotos, las operaciones llevadas a cabo fuera de la unidad presentan falencias especialmente de tipo logístico.
La gestión de la información para planear y ejecutar las misiones debe ser prioridad de la institución.

Identificar las fallas para proveer condiciones minimas de mando, comunicaciones, logística y otros cuando se opera fuera de la unidad. 
3) REALIZACIÓN DEL PRODUCTO (EJECUCIÓN DE OPERACIONES AÉREAS) DEL COMANDO DE COMBATE: Comprende el eje del cumplimiento de la misión, ya que corresponde a la ejecución de las operaciones aéreas del Comando de Combate. El cumplimiento de las condiciones previas antes sugeridas para la implantación del sistema de calidad supone la correcta ejecución del proceso y por consiguiente el éxito en el cumplimiento de la misión.

\section{4) MEJORA CONTINUA: APLICACIÓN DE PROGRAMA} DE MEJORAMIENTO CONTINUO PARA EL SISTEMA DE

CALIDAD: La propuesta para aplicación del programa de mejoramiento continuo sugiere la utilización de mecanismos recomendados para la implantación de un programa de mejoramiento continuo, utilizando principalmente los mecanismos basados en el mejoramiento de las observaciones halladas durante la investigación (ver Cuadro 2).

\section{HALLAZGO}

\section{RECOMENDACIÓN}

\section{CULTURA DE CAIIDAD}

Creencias y suposiciones del personal influyen en la percepción que tienen de la tarea y los objetivos a cumplir, distorsionando ciertos aspectos del cumplimiento de la misión.
Se debe implantar un programa de educación en filosofía de calidad y cultura de calidad en el que participe toda la organización.

\section{ESTANDARES DEOPERACION}

El Grupo de Combate busca racionalizar la planta de pilotos, concentrándola en una dependencia, facilitando el desempeño y la proyección del personal.

Los comandos han aumentado el número de pilotos en el Grupo de Combate a casi $50 \%$ de la planta total de la unidad.

La ejecución de operaciones desde la unidad ofrece ventajas sobre las que son lanzadas desde otras diferentes.

No necesariamente todos los aspectos de ejecución de operaciones son mejores cuando se opera desde la base habitual.

Existe una herramienta para adelantar entrenamiento de misión llamada Entrenamiento Continuado, la cual es vista positivamente por el personal.

Los programas de entrenamiento y las operaciones se complementan pero también se interfieren.

A pesar de la interferencia entre el vuelo y la oficina, la carrera administrativa debe ser complemento de la de piloto.

CUADRO 2: RECOMENDACIONES PARA IMPLANTAR UN PROCESO DE MEJORAMIENTO CONTINUO
Seguir adelante con estas iniciativas, impulsándolas y completando planes de expansión del grupo que se identifican plenamente con la implantación del sistema de calidad.

Ante la necesidad de operar desde otras bases aéreas, proveer el soporte mínimo necesario para despliegue de aéreos avanzados.

Reestructurar la organización de la unidad para evitar interferencias de tipo administrativo en los pilotos al operar desde la base.

Implementación como complemento de lo ya establecido con apoyo de la gerencia para colmar las necesidades de entrenamiento de misión.

Se debe tener en cuenta la situación actual y las necesidades operativas para balancear el entrenamiento y no ponerlo por encima de la misión, ni lo contrario.

Adoptar partes de modelos estudiados (benchmarking). Proponer a todos las niveles de mando la estrategia de "desarrollo de carrera" lo que permite concentrar en ciertos grados el grueso de la responsabilidad operacional.
Utilizar mecanismos adicionales para el establecimiento de niveles de calidad de operación de acuerdo a las normas utilizadas como referencia (ISO 9001:2000 principalmente), es factor importante a tener en cuenta en la orga- nización si se desea lograr niveles de desempeño más allá del alcance del presente escrito. Según lo consideren los respectivos comandantes, estos mecanismos pasan de ser importantes a indispensables. 